\title{
FROM FUNDAMENTAL BRAIN TUMOR SCIENCE TO INTERDISCIPLINARY BEDSIDE CARE; THE OUTCOME REPORT FROM THE NEURO-ONCOLOGY SCIENTIFIC CLUB SECOND MEET- UP (NOSC-2), 19th APRIL 2012, MASHHAD, IRAN
}

\author{
ANVARI K. ${ }^{1}$, BAHADORKHAN G.H. ${ }^{2}$, SILANIAN-TOUSSI M.1, RAHIGHI S.4, GHAVAMNASIRI M.R. ${ }^{1}$, \\ SAEEDI M. ${ }^{2}$, MOJARRAD M. ${ }^{2}$, TABATABAEE YAZDY S.A. ${ }^{2}$, NEKOOEE S. ${ }^{2}$, NOWFERSTI G.H. ${ }^{1}$, SALEK R. ${ }^{1}$, \\ MASHHADI NZHAD H. ${ }^{2}$, TAGHZADEH KERMANI A. ${ }^{1}$, HOMAEI-SHANDIZ F. ${ }^{1}$, ALEDAVOOD S.A. ${ }^{1}$, \\ EHSAEI M.R. ${ }^{2}$, FARAJI-RAD M.2, RAFATI A.R. ${ }^{2}$, ABILI M.2, BAHAR VAHDAT H. ${ }^{2}$, SAFAIE YAZDI A. ${ }^{2}$, \\ BIDOYEI F. ${ }^{1}$, KHOSHROO F. ${ }^{1}$, VARSHOEE TABRIZI F. ${ }^{1}$, FANI PAKDEL A. ${ }^{1}$, SHAHID SALES S. ${ }^{1}$, \\ MIRSADRAEE M. ${ }^{1}$, YOUSEFI A.H. ${ }^{3}$, HOSSEINI S. ${ }^{1}$, NOORI FARD M. ${ }^{5}$, DEHESTANI M. ${ }^{5}$, MIRSHAHI J. ${ }^{5}$, \\ MUKHOMOROVA L. ${ }^{7}$, AFARID M. ${ }^{6}$, HEJAZI-FARAHMAND S.A.R. ${ }^{6}$, TORABI-NAMI M. ${ }^{7}{ }^{*}$
}

\author{
${ }^{1}$ Cancer Research Center, Omid Hospital, Faculty of Medicine, Mashhad University of Medical Sciences, Mashhad, Iran \\ ${ }^{2}$ Faculty member, Mashhad University of Medical Sciences, Mashhad, Iran \\ ${ }^{3}$ NOSC member, Mashhad, Iran \\ ${ }^{4}$ NOSC Lead Faculty, Mashhad, Iran \\ ${ }^{5}$ NOSC member, Mashhad, Iran \\ 6Behphar Group, Tehran, Iran \\ ${ }^{7}$ Medical division, Behphar Group, Tehran, Iran \\ *Corresponding Author: Email- m_torabi@behestandarou.com
}

Received: May 22, 2012; Accepted: June 25, 2012

\begin{abstract}
The second session of the interval meetings of the Neuro-Oncology Scientific Club (NOSC-2)- Mashhad, took place on 19th April 2012 in Mashhad, Iran. NOSC is an interdisciplinary forum providing updates in brain tumor diagnosis and management. The focus for this scientific meeting was how fundamental brain tumor science can foster clinical advances in treating patients with glial brain tumors and glioblastoma multiforme in particular. Having experts from radiation oncology, neurosurgery, neuroradiology, pathology, neurology and medical genetics under one roof, turned this meeting to a touching platform for redirecting debates while putting brain tumor patients' survival and quality of life first. This is expected to lead to provincial and later countrywide practice guidelines for interdisciplinary management of brain tumors. Translational research, bridging fundamental science to bedside care for brain tumors is also on schedule for NOSC activities. During the "NOSC-2" meeting, current brain tumor international guidelines were reviewed to ensure compatibility to our local setting and practice. Furthermore, strategies for brain tumor patient referral and bidirectional collaboration between various allied disciplines were lined up. Research opportunities in basic science and clinical neuro-oncology within Mashhad University of Medical Sciences cancer research center were shared and targeted. The current draft of the provincial brain tumor registry (PBTR) software was presented. PBTR was agreed to be installed in two distinguished oncology centers in Mashhad as pilot. Taking initial steps towards provincial practice guideline definition (primarily for high grade gliomas) will be the main agenda constituent for the upcoming NOSC session to be held in mid September 2012. Keywords- Brain tumor, interdisciplinary care, translational research, NOSC, guideline definition group, registry, Mashhad
\end{abstract}

Citation: Anvari K., et al. (2012) From Fundamental Brain Tumor Science to Interdisciplinary bedside Care; the Outcome Report from the Neuro-Oncology Scientific Club Second meet-up (NOSC-2), 19th April 2012, Mashhad, Iran. International Journal of Medical and Clinical Research,ISSN:0976-5530 \& E-ISSN:0976-5549, Volume 3, Issue 5, pp.-168-175.

Copyright: Copyright@2012 Anvari K., et al. This is an open-access article distributed under the terms of the Creative Commons Attribution License, which permits unrestricted use, distribution and reproduction in any medium, provided the original author and source are credited. 


\section{Introduction}

Interdisciplinary approach is proven to cater benefits in better management of cancer including brain tumors [1]. Therefore, crossing the linkage between different discipline experts who actively involve in management of brain tumors is believed to be crucial. This gave rise to our Neuro-Oncology Scientific Club's establishment. The Neuro-Oncology Scientific Club (NOSC) is a scholarly forum for sharing updates in brain tumor science and strategizing how together serve brain tumor patients better. This scientific club has been established since 2011. NOSC has provincial and central steering boards in Iran, in cities like Tehran, Mashhad, Isfahan, Shiraz and Tabriz.

NOSC continues to hold interval meetings to pursue its aims which focus on applying shared international and local experience to improve brain tumor patients' survival and quality of life. After two successful sessions in Tehran[2] and Mashhad [3], the second Mashhad NOSC meet up was hold on the 19th April 2012 in Mashhad. This meeting hosted neurooncology experts from Khorasan province. Attendees were professionals from neurosurgery, radiation oncology, radiology, pathology, neurology and applied science fields including medical genetics, pharmacology and clinical neuroscience [appendix 1].

This report summarizes the main scientific insights communicated as well as strategic decisions made during round table and plenary discussions. The theme of the second Mashhad- NOSC (MHDNOSC 2) was bridging fundamental brain tumor science and the interdisciplinary bedside care. To cement the gap between everadvancing science in brain tumor and the bedside care, targeting translational-bidirectional research was what pursued during the meeting.

The keynote speakers focused on issues like applying international brain tumor guidelines to our local practice, pitfalls in maximal safe resection of anaplastic gliomas and glioblastoma multiforme (GBM) and the role of neurology in neurooncology. Furthermore, exploring how basic research (genetics-immunology) insights boost success achievement in treating glial brain tumors and mainly high grade gliomas (HGG), was an integral part of the agenda.

One of the aims of NOSC's current and forthcoming activities is to launch the prepared software for brain tumors registry purpose. The draft of this software had been refined after inputs from NOSC steering committees in different provinces. This software draft which can be employed as an efficient tool in careful registration of our brain tumor patients' data, was presented, discussed and agreed about during the meeting.

\section{Applicable Clauses of International Brain Tumor Guidelines to Our Local Practice}

The two widely accepted and referenced international guidelines in brain tumors are the National Comprehensive Cancer Network (NCCN) and the European Society for Medical Oncology (ESMO) practice guidelines. We selectively reviewed the latest versions of these two guidelines on high grade malignant gliomas [4,5]. Our aim was to define which clauses best comply with our local practice setting in diagnosis, treatment and follow up HGGs. Both guidelines emphasize on the multidisciplinary approach towards brain tumor management $[4,5]$.

\section{Diagnosis}

ESMO classifies malignant giomas to glioblastoma (WHO grade IV), anaplastic astrocytoma (WHO grade III), mixed anaplastic oligoastrocytoma (WHO grade III) and anaplastic oligodendroglioma (WHO grade III) $[4,6]$.

\section{Molecular Markers}

There are molecular markers such as genetic loss on chromosomes $1 p / 19 q$ which predict higher chemosensitivity of glial brain tumors.

Patients with brain tumor having $1 p / 19 q$ deletion are expected to have a prolonged natural history irrespective of the treatment Assessment of these deletions are recommended to support diagnosis of oligodendroglioma [7]. NCCN supports $1 p / 19 q$ analysis for prognosis assessment in anaplastic gliomas [5].

In practice we see GBM patients more favorably responding to concomitant chemoradiotherapy and adjuvant chemotherapy with Temozolomide (TMZ). It has been documented that epigenetic silencing of the methyl-guanine methyl transferase (MGMT) gene promoter by methylation (i.e. methylated MGMT status) partially prevents tumor to have the chemotherapy induced DNA damage repaired [8]. However, due to lack of standard and reproducible method, routine check for MGMT methylation status is not warranted. Moreover, the current practice is short of alternative treatment for unmethylated MGMT GBMs, therefore the idea of MGMT methylation status determination should be kept within clinical trials $[4,5,7]$.

\section{Staging}

For glial brain tumors staging, neuroradiological, surgical and pathological evaluations are pivotal. Magnetic resonance brain imaging (MRI) plus the extent of resection and determination of residual disease in 24-48 hours after surgery, also the histopathological findings would assist staging. Lumbar puncture is generally not useful and guidelines do not support the need for staging of other organs [5].

\section{Prognosis}

Patients with lower tumor grade, radical tumor resection, younger age (i.e. less than 50 years) and favorable performance status with preserved neurological functions would generally have a better prognosis [9-11]. In recursive partitioning analysis (RPA), patients with lower RPA class (i.e. III and IV) have good prognosis and benefit the maximal outcome from current treatment of choice (concomitant chemoradiotherapy and adjuvant chemotherapy with $\mathrm{TMZ}$ ) compared to those in RPA class V $[12,13]$. The performance status has gained special attention in defining prognosis [--]. Stratification is based on karnofsky Performance Status (KPS) score. For poorly performing patients (KPS $<70$ ), functional external beam RT (standard or hypofractionated), chemotherapy or best supportive care is suggested [4].

\section{Treatment Plans}

The use of steroids (e.g. dexamethasone $8-16 \mathrm{mg}$ ) is to control tumor associated edema. Steroids should be rapidly tapered and are not required in those who do not suffer from increased intracranial pressure and neurological deficits. Following tumor resection and optimal radiotherapy-chemotherapy and in absence of 
above mentioned conditions, the prolonged use of steroids is by no means indicated [5]. Based on NCCN, corticosteroids may not be needed in asymptomatic patients and if started, $\mathrm{H} 2$ blockers should be used with them in high risk patients for GI complications [4].

Since endocrine disorders are quite frequent in these patients, evaluation of pituitary- adrenal axis, gonadal and thyroid function is worthwhile [4].

Depression, other neuropsychiatric disorders and deep vein thrombosis (DVT) should be timely addressed by allied disciplines' professionals $[4,5]$

In patients primarily present with seizure, antiepileptic (AED) therapy should be sought $[5,14]$. On the other hand, prophylactic AED before or after surgery is not recommended $[4,5,14]$. Instead of using first generation AED which strongly induce hepatic enzymes and interfere with many chemotherapeutic agents (but not TMZ), the use of third generation agents such as Lamotrigine and Pergabalin are preferred $[5,14,15]$. This can be re-emphasized for working teams in brain tumor management as optimal regimens lead to more favorable outcomes.

\section{Newly Diagnosed Glial Brain Tumors}

a. Surgical interventions aim at maximal safe resection if feasible. The use of chemotherapy wafers (Carmustine wafers) in the surgical cavity prior to chemotherapy is shown to marginally improve survival. The maximal safe resection with or without BCNU (Carmustine) wafers placement is a category 2B recommendation from NCCN [4]. However, no evident data comparing the wafer implants with standard TMZ/RT is available and there is no strong evidence supporting the big additive value of Carmustine implants[16-18].

b. Radiotherapy should be fractionated and focal (60 Gy, 30-33 fractions of 1.8-2 Gy). RT dose is decreased to $40 \mathrm{~Gy}$ in 15 fractions in elderly. Escalating the dose beyond $60 \mathrm{~Gy}$ is not of value.

c. The standard of care for GBM treatment (age $<70)$ is chemoradiotherapy followed by adjuvant chemotherapy with TMZ. In concurrent chemoradiotherapy, TMZ is administered 7 days a week for 6 weeks. The dose is $75 \mathrm{mg} / \mathrm{m}^{2}$ and istaken $1-1.5$ hours before radiotherapy. This phase is followed by 4 weeks of break and at least 6 cycles of adjuvant TMZ (5/28 days) at the dose of $150-200 \mathrm{mg} / \mathrm{m}^{2}$. P.carinii prophylaxis with Trimethoprim-Sulfomethoxazole is given during the concurrent phase. Absolute Neutrophil Count (ANC) and platelet count should be checked and TMZ dose should be adjusted or temporarily suspended as per prescribing information $[4,5]$.

Based on the current evidence $[19,20]$, guidelines do not support adjuvant chemotherapy with Procarbazine, Lomustine and Vincristine (PCV regimen) in grade III and IV tumors of unfavorable expected outcome. For the GBM adjuvant therapy with TMZ, stratification depends upon treatment with BCNU whatever the strata, mapping is according to KPS $(\geq$ or $<70)$. As per current guidelines, for those treated with $B C N U$ wafers and KPS $\geq 70$, fractionated external beam RT \pm concomitant and adjuvant TMZ should be considered (category 2B) [4]. For whom not treated with BCNU wafers, based on KPS score (i.e. $\geq$ or $<70$ ), concurrent chemoradiotherapy and adjuvant chemotherapy with TMZ is recommended by category 1 and $2 B$, respectively [4].

\section{Recurrent Disease}

Anaplastic Astrocytoma (AA) is more likely to respond to TMZ chemotherapy. For patients who progress after previous chemotherapy, no established chemotherapy regimen is available and patients are best treated within investigational treatment protocols. In recent studies, Bevacizumab ( \pm Irinotecan) is shown to provide high imaging response and steroid sparing $[5,21]$. However, vascular permeability changes has prevented these effects to be long lived. The effect of these investigational protocols on life expectancy is yet unclear.

Generally, when recurrence is diffuse or multiple, guidelines recommend best supportive care when PS is poor. Systemic chemotherapy or surgery is supported for symptomatic large lesions [4]. In case patients had shown good response to previous RT, reirradiation can be considered $[4,22]$.

Post operative MRI within 72 hours is recommended to ensure the extent of resection [4]. Although MRI has remained the standard imaging method, contrast enhancement and sometimes falsely presumed tumor progression on imaging 4-8 weeks after the end of radiotherapy is misleading. This may be due to the reactive process following RT. Nevertheless, this may be true progression. Therefore, re-imaging with second MRI 4-8 weeks later is recommended. For GBM, taking MRI 2-5 weeks after RT, then each 2-4 months and then less frequently is recommended $[4,23,24]$. To delineate pseudoprogression from true progression earlier, stateof-the-art imaging techniques should be employed. These include magnetic resonance spectroscopy (MRS), diffusion weighted MRI (DW-MRI), Single-photon emission computed tomography (SPECT) and positron emission tomography (PET). All of the above except PET are currently available in Iran [2].

\section{Pitfalls in Maximal Safe Resections of HGGs (Clues for Suc- cess)}

\section{High Grade Glioma Challenges}

Glial tumors may develop almost anywhere in CNS but are most frequently seen in cerebral hemispheres, optic pathways and brain stem $[25,28]$. They vary in malignant behavior and are generally classified as low and high grade gliomas. High grade gliomas (HGG) typically have more consistent growth speed and symptoms with inferior outcome compared to low grade gliomas [26]. Central brain tumor registry of the United States (2010) reported that gliomas comprise $46 \%$ of primary adult brain tumors. Almost $20 \%$ of all adult CNS gliomas are shown to be low grade [27]. In HGGs, glioblastoma multiforme (GBM) is known as the most frequent brain tumor $(25 \%)$ which rapidly progresses and can present as primary (de novo) or secondary (progressed LGG to GBM). GBM is an aggressive tumor which can typically invade corpus callosum and involve both hemispheres (Fig.1).

Upon presentation, patients have symptoms pertaining to either the location of the tumor or increased intracranial pressure. These symptoms include weakness, ataxia, seizure, headache, focal neurological deficits, cognitive impairments and beyond [28].

\section{Brain Tumor Surgery; Helping not Harming}

Surgical resection of brain tumors can be done immediately (large mass, extensive symptoms) or delayed (small mass, minimal symptoms). Following resection,careful clinical and radiolographic surveillance should be sought. Subsequent resection may be 
required when we confront a progressive mass or symptoms [29]. The goal is maximal safe resection when feasible, especially when patients are symptomatic or presumed diagnosis is unclear. Although there are scant prospective randomized data, retrospective reviews report improved outcome with earlier and more extensive resections [30,31].

Craniotomy and brain surgery put the patients at risk for new neurological deficits. Eloquent brain areas may be damaged when functional surgery is not optimally possible. The risk of new neurological deficits after craniotomy ranges from $10-25 \%$ in modern surgical setups [32]. The factors which expose the patients to higher risk include but restricted to age $>60$, KPS $<60$, deep tumor location and tumor proximity to eloquent brain areas. Data have indicated that gross total resection (GTR) of intrinsic brain tumors is associated with fewer neurological deficits as compared to subtotal resection $[30,32]$. Neurological complications are mainly due to direct injury to normal brain, brain edema, vascular injury and hematoma. To avoid these complications, brain mapping for surgical planning should be done. Identification of tumor limits during surgery allows less damage to functionally important cortical and subcortical brain areas. To achieve the goal as per pathology and to make maximal tumor resection without causing significant longterm morbidity, preoperative monitoring should be considered [33].

\section{Brain Mapping for Presurgical and Intraoperative Planning}

There are invasive (electrocorticography and Wada test) and noninvasive (functional MRI, PET, magnetoencephalography, DWI and Diffusion Tensor Imaging) measures available for the above purpose. For intraoperative mapping and monitoring (awakefunctional surgery), motor cortex stimulation and somato-sensory evoked potentials (SSEP) are applicable. These setups are being initially installed in designated centers in Iran.

Minimally invasive intraoperative auxiliary tools such as frameless stereotaxis and magnetic navigation systems would help surgeons to identify and localize residual tumor tissue. After all, it is plausible that total resection is fully practical due to highly infiltrative nature and indefinite borders of HGGs [32,33].

We are trying to soon apply intra-operative navigation and cortical mapping techniques to our setting, hoping to optimize maximal safe resection in brain tumors.

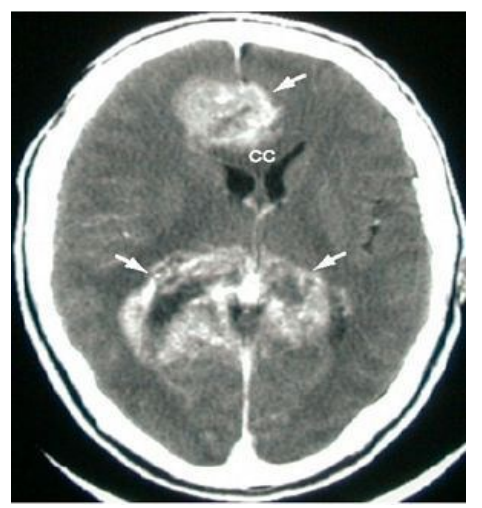

Fig. 1- GBM is an aggressive tumor which often crosses the midline invading along the whole length of corpus callosum (cc). This figure demonstrates a typical butterfly pattern (arrows). Adapted from [28].

\section{Neurology and Brain Tumor Management}

Given the strong interdisciplinary character of neuro-oncology, active contribution of allied disciplines should be encouraged [34]. Beside neurosurgery, radiation oncology, radiology and pathology, neurology can play an integral role in management of brain tumor patients. Sign and symptom guided detection and follow up of tumoral or metastatic brain lesions, neurophysiological assessments, CSF analysis, management of paraneoplastic disorder, seizure, pain, paresis, sleep problems, headache, etc is expected to be rendered in neurology [35].

Many antineoplastic drugs cause neurotoxicity, thus a close collaboration between radiation/medical oncologists and neurologists would be required. Here, we outline the optimized use of antiepileptic drugs (AEDs) as an exemplary issue for which neurology contributes to neuro-oncology. Seizure is a common manifestation seen in brain tumor patients and AEDs are very frequently used in these patients [36]. The risk of side effects and drug-drug interactions (DDI) make the proper AED selection a major concern. The use of classical AEDs such as Phenobarbital (PB), Phenytoin (PHT) and Carbamazepine (CBZ) has led to increased interactions. The concomitant chemotherapy efficacy can be largely affected by these interactions. While enzyme inducing AEDs (EIAEDs) diminish therapeutic levels and hence the efficacy of cytotoxic drugs (CTDs), AEDs with enzyme inhibitory property (Valproic Acid-VPA) may lead to bone marrow suppression and other toxicities related to decreased CTDs' metabolism. Moreover, the existing signs of brain damage secondarily to tumor or previous neurosurgery, radio- or chemotherapy can markedly add to the neurotoxicity of AEDs' neurotoxicity.

The spectrum of neurotoxicity includes cognitive dysfunctions which result in diminished KPS in brain tumor patients [36].

\section{Drug-Drug Interactions; a Major Concern}

For the following reasons treatment of cancer patients puts them at high risk for drug-drug interactions:

- They receive multiple medications (cytotoxic chemotherapy, hormonal agents and palliative care drugs).

- They very often receive medications for underlying disease conditions such as neurological or cardiovascular diseases.

- They often suffer from disease of medication induced hepatic or renal dysfunction.

The potential risk for DDI in brain tumor patients rises from $50 \%$ to $100 \%$ when the number of medications increases from five to seven.

When using AEDs, DDI may affect patients chemotherapy outcome. Therefore individualized care should be taken to avoid inappropriate AED regimens. The induction of cytochrome P450 (CYP450) by EIAEDs can drastically affect CTDs' metabolism. Therefore this class of AEDs should be avoided in brain tumor patients receiving chemotherapy. From common brain tumor chemotherapeutic agents, TMZ is not shown to have noticeable DDI with commonly used AEDs.

Table 1 summarizes major known AEDs-CTDs interaction. (Table 1)

Based on discussed notions, selecting non- EIAEDs (newer AEDs) are preferred. These include Lamotrigine, Gabapentine, Pregabaline, etc.

On the other hand, if a CTD inhibits hepatic enzymes, toxic levels 
of the AEDs will become a concern. In case the CTD induces the hepatic microsomal system, it facilitates AED clearance, thus the risk of seizure is increased [36-66].

Table 1-Demonstrates the common drug-drug interactions in neuro-oncology practice. Many of these medications are concomitantly used in brain tumor patients.

\begin{tabular}{|lll|}
\hline Group CTD & Type of CTD & AED that Reduces CTD \\
& Busulphan & PHT [37] \\
Alkylating Agents & Cyclophosphamide & PB, PHT [38,39] \\
& Ifosfamide & PHT [39] \\
& Thiotepa & PHT [40] \\
& Temozolomide & None [15] \\
Antimetabolites & Methotrexate & PB, PHT, CBZ [41,42] \\
& 9-Amino camptotecin & PB, PHT, CBZ [43,44] \\
& Irinotecan & PB, PHT, CBZ, PRM, OXC [45,47] \\
& Topotecan & PHT [48] \\
Antimitotic Agents & Paclitaxel & EIAEDs [49-51] \\
& Teniposide & PB, PHT [52] \\
& Etoposide & AEDs [53] \\
& Vincristine & PHT, CBZ [54] \\
& Bortezomib & EIAEDs [55] \\
& Enzastaurin & EIAEDs [56] \\
& Erlotinib & EIAEDs [57,58] \\
& Gefitinib & PB, PHT, CBZ, PRM, OXC [59] \\
Imatinib & PHT, CBZ, PRM, OXC, TPM [60-62] \\
Proteinkinase & Sorafenib & EIAEDs [63] \\
Inhibitors & Tipifarnib & PB, PHT, CBZ [63] \\
& Vatalinib & PB, PHT, CBZ, PRM, OXC [64] \\
& Temsirolimus & PB, PHT, CBZ, OXC [65,66] \\
& Sirolimus & PB, PHT, CBZ, OXC [65,66] \\
& Everolimus & EIAEDs [65,66] \\
\hline & &
\end{tabular}

AED: antiepileptic drug; EIAED: enzyme-inducing antiepileptic drug; CTD: chemotherapeutic drug; CBZ: carbamazepine; OXC: oxcarbazepine; PB: phenobarbital; PHT: phenytoin; PRM: primidone, TPM: topiramate;

\section{Corticosteroid use in Brain Tumor Patients}

Corticoseroids lower the intracranial pressure primarily in vasogenic edema induced by space occupying lesions such as tumors. This is due to steroid effects on vascular permeability and direct normalizing effect on endothelial cell function. Dexamethasone in high doses is shown to dramatically reduce edema surrounding the brain tumor and metastases.

Dexamethasone caters its beneficial effects through rapid closure of blood brain barrier which is disrupted due to tight junction dysfunction and fenestrated endothelium leading to edema.

The tumor surrounding vasogenic edema presents in digitiform appearance in brain imaging, while cytotoxic edema lacks this feature. Fig. 2 demonstrates how vasogenic versus cytotoxic edema can appear different (Fig. 2).

Taking shared decisions to commence, continue, hold or discontinue steroids and AEDs will be possible when an interdisciplinary approach is in place. As a part of the multifaceted teamwork in management of brain tumor, the role of neurology should be further emphasized.

\section{Towards Translational Brain Tumor Research in NOSC}

Basic researches on CNS cancers are carried out in several fields. Identifying appropriate biomarkers is one of the important objectives of these studies. These experiments aim at identifying either diagnostic or prognostic biomarkers.
The use of MGMT promoter methylation status assessment to predict glial brain tumor response to chemotherapy with Stupp protocol with TMZ, is an exemplary successful application of basic science research to define prognosis in CNS cancer treatment. Identifying molecular tools for the treatment of brain tumors is another interesting area of basic research on CNS tumors. One of interesting approaches in the treatment of brain cancers is immunotherapy. This approach involves the use of tools such as DNA vaccines, tumor-associated antigens, especially cancer/testis antigens to stimulate the immune system against tumor cells. Fig. 3 illustrates a cancer-immunology interface technique known as Serological Expression Cloning (SEREX). This is used for identification of human cancer antigens which are the targets for immunotherapy (Fig.3).
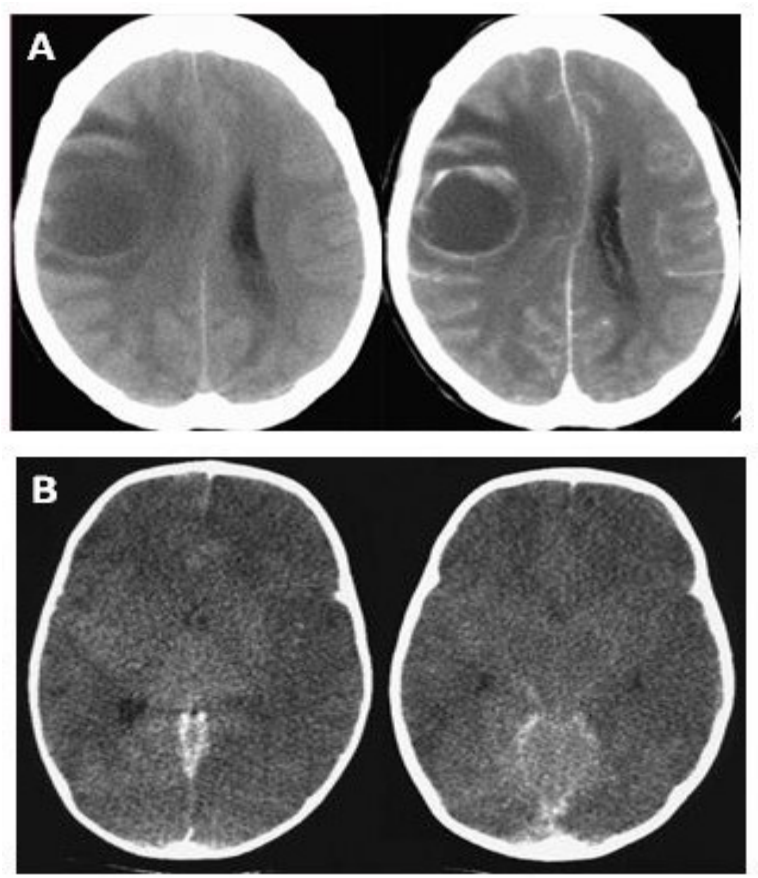

Fig. 2- Distinct difference in vasogenic versus cytotoxic edema appearance in brain imaging. A: 57 year old female with headache and tongue deviation, demonstrating digitiform edema surrounding a mass in right fronto temporal lobe. B: generalized hazy pattern with enclosed cisterna, compatible with cytotoxic edema.

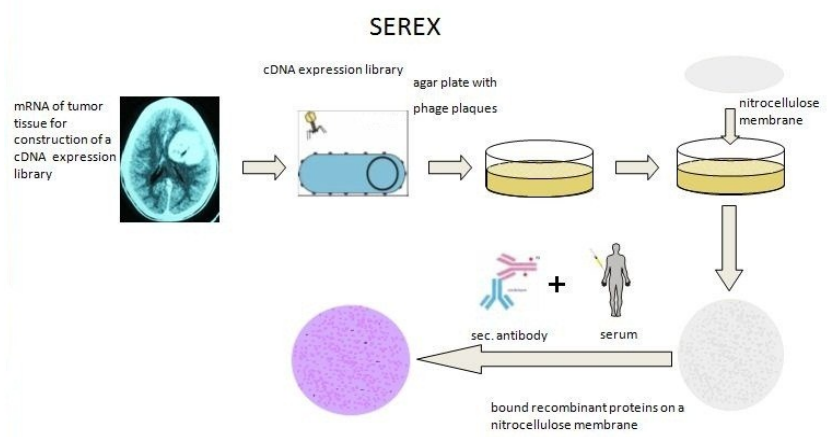

Fig. 3- Identification of tumor associated antigens in intracranial tumors. mRNA: messenger RNA, cDNA: complementary DNA, SEREX: Serological Expression Cloning 
Over past few decades there has been series of investigations on the use of genetically modified vectors (such as viral carriers) to correct genetic defects in tumor cells and eventually to control proliferation of malignant cells or to induce programmed cell death (apoptosis).

The use of micro-RNA molecules to manipulate tumor cell gene expressions provides a new horizon towards the treatment CNS tumors $[67,68]$.

As an interdisciplinary working team on treatment of brain tumor, we discussed the possibility to set up some of the above research protocols in our cancer research center. The research questions will be addressed and we will come up with the first translational brain tumor research proposal for discussion during the next NOSC meeting in Mashhad.

\section{Provincial Brain Tumor Registry, Khorasan Province, Mashhad Univ. of Medical Sciences}

One of the main objectives that Mashhad NOSC pursued since the opening session, was to define a clinical data gathering system to help registering brain tumor patients across khorasan Province. We have taken steps to develop contents for a software to categorize our brain tumor patients' data in a useful, accurate and ordered way. This will help us to assemble clinical information in a uniformed setting. By this, brain tumor data can be integrated, processed and forwarded to project managers for clinical or translational researches which are being designed in Cancer Research Center in Mashhad University of Medical Sciences. The pre-final draft of this software was demonstrated during Mashhad $2^{\text {nd }}$ NOSC meeting. This draft has gained agreement from experts opinion in different provinces, however needs to further align with the cancer registry requirements of the ministry of health in Iran. The final version preparation is in progress.

This data registry software which is introduced as PBT (Provincial Brain Tumor Registry) contains the followings sections: 1) The patient's clinical information section for demographic and disease condition details, 2) Presenting complaints and symptoms 3) Brain tumor information, including the location and histological properties, 4) Imaging data, with the possibility of image storage 5) CSF analysis, 6) Pathology reports, 7)Surgery data for extent of resection and margin status 8) Chemoradiotherapy section for dose, duration and experienced side effects, 9) outcome section, for clinical response and survival data and 10)Post mortem data section.

Mashhad NOSC panel and faculty decided to let a pilot registry using the demonstrated software in two main oncology centers in Mashhad (i.e. Omid Ocology Hospital and Imam Rezaa Oncology Clinic). This will become effective as of mid July 2012.

\section{Summary}

The NOSC-2 Mashhad meeting provided a forum for neurooncology experts to further come into shared insights and common strategies for more efficient team spirit in brain tumor patients care.

Below points were consolidated upon closing:

- Provincial Brain Tumor Registry (PBTR) software is to be installed in 2 centers as pilots in near future.

- For the next meeting, attendees decided to present past 5 years report of GBM status in Mashhad territory. They also planned to bring along extended survivors' data for discussion.

- Participants re-emphasized the necessity of consistent interdisciplinary collaborations. This can be further be defined in the provincial guideline Mashhad NOSC plans to develop.

\section{Acknowledgement}

Authors would like to thank medical affairs of Behestan Darou P.J.S. for scientific supports, preparations and assistance at this meeting. Special thanks go to Drs. P. Dindoust, M. Pesyan, I. Alekseeva (MSD), R. Fahim (MSD), Sh. Ibrahim (MSD), F. Mohammadzadeh, H. Rouhani, P. Mobasheri for their supports. Warm thanks to all experts from Mashhad University of Medical sciences and cancer research center (MUMS) who participated and contributed to this special discussion forum.

\section{Disclosure}

NOSC (The Neuro-Oncology Scientific Club) is a professional forum for the exchange of experts' experience and updates on brain tumors in an interdisciplinary fashion. NOSC plans to act as a working team and further as a guideline definition group in Khorasan Province, Mashhad and meanwhile has no competing interest to disclose

\section{Other Scientific Contributors}

Drs. Sadeghi Ivari M., Fazl Ersi M., Nosrati F., Dehghan P., Bayat Mokhtari N., Dayani M., Amirabadi A., Agha Mohammadian J., Tajdini Sh.

\section{Funding Support}

This communication in a form of a meeting has been made possible through funding support from Behestan Darou P.J.S.

\section{Notes}

1. Open access is a property of individual works, not necessarily journals or publishers.

2. Community standards, rather than copyright law, will continue to provide the mechanism for enforcement of proper attribution and responsible use of the published work, as they do now.

\section{References}

[1] DeVita V.T., Hellman S., Rosenberg S.A. (2005) Cancer: Principles and Practice of Oncology, 7th ed.

[2] Haddad P., Zali A., Tabatabaeefar M., Nikoofar A., Hadizadeh Kharazi H., Ghadyani M., et al. (2012) NOSC- Report and Opinion, 4(2),42-53

[3] Anvari K., Bahadorkhan G., Nekooi S., Taghizadeh A. Kheradmand H., Nowferesti G. et al. (2011) Webmed Central. Oncology, 2(10).

[4] Brem S.S., Bierman P.J., Brem H., Butowski N., Chamberlain M.C., Chiocca E.A., DeAngelis L.M., Fenstermaker R.A., Friedman A., Gilbert M.R., et al. (2011) J. Natl. Compr. Canc. Netw., 9, 352-400

[5] Stupp R., Tonn J.C., Brada M., Pentheroudakis G. (2010) Annals of Oncology, 21(5), v190-v193

[6] Louis D., Ohgaki H., Wiestler O., Cavenee W.K. (2007) WHO Classification of Tumours of the Central Nervous System. Lyon: International Agency for Research on Cancer. 
[7] Stupp R., Hottinger A.F., Van den Bent M.J. et al., Ann Oncol.,19 (7), vii209-vii216.

[8] Hegi M.E., Diserens A.C., Gorlia T., et al. (2005) N. Engl. J. Med., 352, 997-1003.

[9] Wen P.Y., Kesari S. (2008) N. Engl. J. Med., 359, 492-507.

[10]Buckner J.C. (2003) Semin. Oncol., 30, 10-14.

[11]Laws E.R., Parney I.F., Huang W., Anderson F., Morris A.M., Asher A. (2003) Journal of Neurosurgery, 99(3), 467-473.

[12]Mirimanoff R.O., Gorlia T., Mason W., Van den Bent M.J., Kortmann R.D., Fisher B., Reni M., Brandes A.A., Curschmann J., et al (2006) Journal of Clinical Oncology, 24(16), 2563-2569.

[13]Paravati A.J., Heron D.E., Landsitte D., Flickinger J.C. (2011) J. Neurooncol., 104, 339-349.

[14]Tremont-Lukats I.W., Ratilal B.O., Armstrong T., Gilbert M.R. (2008) Cochrane Database of Systematic Reviews, 2.

[15]Temodal 8 Prescribing Information (2009) Schering Corporation. U.S. Patent Nos. 5,260,291 and 6,987,108.

[16]Bota D.A., Desjardins A., Quinn J.A., Affronti M.L., Friedman H. (2007) Ther. Clin. Risk Manag., 3(5), 707-715.

[17]Albright R.E., Brenemman J.C., Warnick R.J. (2008) J. Neurosur, 108(2), 236-242.

[18]NICE Technology Appraisal (2008) NICE terminates appraisal of carmustine implants for the treatment of recurrent glioblastoma multiforme, 149.

[19]Lee S.M., Barada M., Gebe R. (2010) A randomised trial of temozolomide vs PCV chemotherapy for recurrent malignant glioma (MRC BR12) ESMO.

[20]Stupp R., Hegi M.E., Gilbert M.R., Chakravarti A., J Clin Oncol, 25, 4127-4136

[21]Chi A.S., Norden A.D., Wen P.Y. (2009) Neurotherapeutics, 6 (3), 513-526.

[22]Patel M., Siddiqui. F., Jin J.Y., Mikkelsen T., Rosenblum M., Movsas B., Ryu S. (2009) Journal of Neurooncology, 92(2), 185-191.

[23]Gunjur A., Lau E., Taouk Y., Ryan A. (2011) Journal of Medical Imaging and Radiation Oncology, 55, 603-610.

[24]David Schiff (2005) Principles and Practice of Neuro-Oncology McGraw-Hill. 3, ISBN 10: 0071425152.

[25]The American Association of Neurological Surgeons (2012) http://www.aans.org.

[26]Buckner J.C. (2003) Seminars in Oncology, 30(6), 10-14.

[27]Porter K.R., McCarthy B.J., Freels S., Kim Y., Davis F.G. (2010) Neuro-Oncology, 12(6), 520-527.

[28]Tonn J.C., Westphal M., Rutka J.T. (2010) Oncology of CNS Tumors, Second edition, Springer, 20-26.

[29]Pang B.C., Wan W.H., Lee C.K., Khu K.J., Ng W.H. (2007) Ann. Acad. Med. Singapore, 36(5), 358-63.

[30]Sanai N., Berger M.S. (2008) Neurosurgery, 62(4), 753-764.

[31]Stummer W. (2008) Neurosurgery, 62, 564-576.

[32]Brennan C.W. (2007) A Clinical Approach. First edition, Informa. healthcare, 91-107.

[33]Spena G., Nava A., Cassini F., Pepoli A., Bruno M., D'Agata F., Cauda F., Sacco K., Duca S., Barletta L., Versari P. (2010) Acta. Neurochir., 152(11), 1835-1846.

[34]Easaw J.C., Mason W.P., Perry J., Laperriere N., Eisenstat D.D., Del Maestro R., Belanger K., Fulton D., Macdonald D. for the Canadian Glioblastoma (2011) Current Oncology, 18
(3), e126-e136.

[35] Grisolda W., Heimansb J.J., Postmab T.J., Grant R., Soffietti R. and The Neuro-oncology Panel of the EFNS. (2002) European Journal of Neurology, 9, 201-205.

[36]Benit C.P., Vecht C.J. (2012) Eur. Assoc. Neurooncol. Mag., 2 (1), 15-24.

[37]Hassan M., Oberg G., Bjorkholm M., et al. (1993) Cancer Chemother. Pharmacol., 33, 181-186.

[38]De Jonge M.E., Huitema A.D., Rodenhuis S., et al. (2005) Clin. Pharmacokinet., 44,1135-1164.

[39]Jao J.Y., Jusko W.J., Cohen J.L. (1972) Cancer Res., 32, 2761-2764.

[40]Ducharme M.P., Bernstein M.L., Granvil C.P., et al. (1997) Cancer Chemother. Pharmacol., 40, 531-533.

[41]De Jonge M.E., Huitema A.D., Van Dam S.M., et al. (2005) Cancer Chemother. Pharmacol., 55, 507-510.

[42]Relling M.V., Pui C.H., Sandlund J.T., et al. (2000) Lancet, 6, 285-290.

[43]Riva M. (2005) Neurol Sci., 26 (1), S40-S42.

[44]Grossman S.A., Hochberg F., Fisher J., et al. (1998) Cancer Chemother. Pharmacol., 42, 118-126.

[45]Minami H., Lad T.E., Nicholas M.K., et al. (1999) Clin. Cancer Res., 5, 1325-30.

[46]Gilbert M.R., Supko J.G., Batchelor T., et al. (2003) Clin. Cancer Res., 9, 2940-2949.

[47]Prados M.D., Lamborn K., Yung W.K., et al. (2006) Neuro Oncol., 8, 189-193.

[48]Reardon D.A., Egorin M.J., Quinn J.A., et al. (2005) J. Clin Oncol., 23, 9359-9368.

[49]Zamboni W.C., Gajjar A.J., Heideman R.L. (1998) Clin. Cancer Res., 4, 783-789.

[50]Fetell M.R., Grossman S.A., Fisher J.D. (1998) J. Clin. Oncol., $15,3121-3128$

[51]Chang S.M., Kuhn J.G., Rizzo J. (1998) J. Clin. Oncol., 16, 2188-2194.

[52]Chang J.W., Chang J.H., Park S.C. (2001) Acta. Neurochir., 143, 297-300.

[53]Baker D.K., Relling M.V., Pui C.H. (1992) J. Clin. Oncol., 10, 311-5.

[54]Rodman J.H., Murry D.J., Madden T. (1994) J. Clin. Oncol., 12, 2390-2397.

[55]Villikka K., Kivisto K.T., Maenpaa H. (1999) Clin. Pharmacol. Ther., 66, 589-593.

[56]Phuphanich S., Baker S.D., Grossman S.A. (2005) Neuro Oncol., 7, 177-182.

[57]Kreisl T.N., Kotliarova S., Butman J.A. (2010) Neuro Oncol., 12, 181-189.

[58]Prados M.D., Lamborn K.R., Chang S. (2006) Neuro Oncol., $8,67-78$

[59]Raizer J.J., Abrey L.E., Lassman A.B. (2010) Neuro. Oncol., 12, 87-94.

[60]Prados M.D., Yung W.K., Wen P.Y. (2008) Cancer Chemother Pharmacol., 61, 1059-1067.

[61]Wen P.Y., Yung W.K., Lamborn K.R. (2006) Clin. Cancer Res., 12, 4899-4907.

[62]Pursche S., Schleyer E., von Bonin M. (2008) Curr. Clin. Pharmacol., 3,198-203.

[63]Reardon D.A., Vredenburgh J.J., Desjardins A. (2011) J. Neu- 
From Fundamental Brain Tumor Science to Interdisciplinary bedside Care; the Outcome Report from the Neuro-Oncology Scientific Club Second meet-up (NOSC-2), 19th April 2012, Mashhad, Iran

rooncol., 101, 57-66.

[64]Cloughesy T.F., Wen P.Y., Robins H.I. (2006) J. Clin. Oncol., 24. 3651-3656.

[65]Reardon D.A., Egorin M.J., Desjardins A. (2009) Cancer., 115, 2188-2198.

[66]Galanis E., Buckner J.C., Maurer M.J. (2005) J. Clin. Oncol., 23, 5294-2304.

[67]Kuhn J.G., Chang S.M., Wen P.Y. (2007) Clin. Cancer Res., 13, 7401-7406.

[68]Godlewski J., Newton H.B., Chiocca E.A. and Lawler S.E. (2010) Cell Death and Differentiation., 17, 221-228.

[69]Stupp R., Hegi M.E., Mason W.P., van den Bent M.J, Taphoorn M.J., Janzer R.C., Ludwin S.K., Allgeier A. (2009) Lancet Oncology., 10, 459-466. 